\title{
Hyperglycaemia in COVID-19: improving recognition and management in a single centre
}

\author{
JORDAN WARDROPE, ${ }^{1}$ IONA E MCKENZIE, ${ }^{1}$ NICHOLAS D BARWELL ${ }^{2}$
}

\begin{abstract}
Background: Hyperglycaemia is a recognised complication of COVID-19 disease and is associated with increased morbidity and mortality. Effects are noted in individuals with and without diabetes and potentiated by the use of recognised COVID-19 treatments such as corticosteroids. Early glycaemic control in the inpatient with COVID-19 disease impacts significantly on outcomes.

Methods: A three-phase improvement project evaluated the recognition and management of hyperglycaemia in 120 adult inpatients with COVID-19 disease over a 4-month period. A local guideline and a separate acute care 'bundle' were implemented to improve performance. The main outcomes of the project were evaluated in a repeated crosssectional design; assessing the performance of regular capillary blood glucose monitoring and appropriate treatment of hyperglycaemia where indicated.

Results: Prior to intervention, $\mathbf{7 8 . 6 \%}$ of patients had appropriate capillary blood glucose monitoring and no patients were deemed to receive appropriate treatment. Following interventions, $83-100 \%$ of patients had appropriate monitoring and $75-100 \%$ received appropriate treatment. Conclusions: In this setting, implementation of a guideline and a care bundle contributed towards improved recognition and management of hyperglycaemia in patients with COVID19 disease. Future study could assess the impact of interventions on a larger scale whilst investigating variation in the subtype of diabetes, patient sex and other demographics on outcomes such as length of stay, morbidity and mortality. Br J Diabetes 2021;21:237-240
\end{abstract}

Key words: COVID-19; diabetes mellitus; hyperglycaemia; glycaemic control; quality improvement

Acute Assessment Unit, Forth Valley Royal Hospital, Larbert, Scotland, UK Diabetes \& Endocrinology Department, Forth Valley Royal Hospital, Larbert, Scotland, UK

Address for correspondence: Dr Jordan Wardrope Acute Assessment Unit, Forth Valley Royal Hospital, Stirling Road, Larbert FK5 4WR, Scotland, UK

Tel: 01324566000

E-mail: jordan.wardrope2@nhs.scot

https://doi.org/10.15277/bjd.2021.324

\section{Background}

There is increasing recognition of the morbidity and mortality associated with the secondary sequelae of COVID-19 disease. In people with and without diabetes, hyperglycaemia is a recognised complication and may have clinically significant effects including diabetic ketoacidosis and hyperosmolar hyperglycaemic state. ${ }^{1}$ The mechanism for COVID-19-associated hyperglycaemia is not yet clear, although likely implicates increased reactive oxygen species production and circulating interleukin-6, along with a state of increased insulin resistance. ${ }^{2}$ There is perhaps a direct link between hyperglycaemia and the physiological response to the stressor of an acute severe viral syndrome - so-called stress hyperglycaemia. Furthermore, the development of hyperglycaemia may be exacerbated or precipitated by some therapies approved for COVID-19 disease, in particular dexamethasone.

It is estimated that hyperglycaemia occurs in around $50 \%$ of patients with COVID-19 during the acute phase of illness. ${ }^{3}$ Hyperglycaemia and/or diabetes in COVID-19 patients are independent risk factors for prolonged hospital stay, critical illness and mortality. ${ }^{4}$ Additionally, it has been reported that controlled blood glucose levels during the first 24 hours of disease correlate with a lower risk of severe disease progression and lower mortality by day 20 of illness in both subjects with and without diabetes. ${ }^{3}$ This highlights the importance of early recognition and intervention in hyperglycaemia.

The pathophysiological response to COVID-19 disease has an impact on which therapies can be used in the acute phase of illness and makes selection of a specific agent more challenging. Current evidence recommends use of insulin therapy in hospitalised and critically unwell patients; ${ }^{2}$ advantages include relatively easy dose titration and the option of an insulin infusion for more challenging cases. Less favourable options include glucagon-like peptide 1 (GLP1) analogues, ${ }^{2}$ sodium-glucose co-transporter 2 (SGLT-2) inhibitors, ${ }^{2}$ thiazolidinediones ${ }^{2}$ and sulfonylureas. ${ }^{1,2}$ Evidence is limited for dipeptidyl peptidase-4 (DPP-4) inhibitors. ${ }^{5}$ Metformin has been associated with a lower risk of death in hospitalised COVID-19 patients ${ }^{6}$ and should be continued in individuals already established on the drug; however, other studies discourage its use in patients with critical disease severity ${ }^{2}$ due to association with lactic acidosis in such disease states. The pharmacological management of hyperglycaemia in COVID-19 is complex and clinicians must adapt their approach accordingly - hence the importance for guidelines and protocols in this relatively novel patient group.

During the emergence of COVID-19 in early 2020, a number of cases of hyperglycaemia in patients with the disease were noted 
by the authors; in particular, in patients with co-existing diabetes and/or those who were receiving dexamethasone therapy. This observation encouraged a search of the relevant literature and guidance available at the time, which was found to be relatively scarce. Given the recognised complications of hyperglycaemia in unwell patients and the associated morbidity and mortality of it alongside COVID-19, the topic was studied further at a local level. This project aimed to evaluate and improve the quality of monitoring and management of hyperglycaemia for hospitalised patients with COVID19 disease.

\section{Methods}

\section{Population}

This project collected data relevant to medical inpatients with a laboratory-confirmed diagnosis of COVID-19 disease within Forth Valley Royal Hospital between November 2020 and March 2021. The population group included adult inpatients within the acute medicine and general medicine wards of the hospital (age range 19-92). Children, obstetric and critical care unit patients were not included in this study.

\section{Data collection}

This study consisted of three periods of data collection by means of a repeated cross-sectional design. Data were collected by two of the authors by examining patient notes, inpatient prescriptions and capillary blood glucose $(\mathrm{CBG})$ monitoring charts. The main outcomes evaluated in the project were performance of regular CBG monitoring and appropriate treatment for hyperglycaemia where indicated.

During each phase of data collection, patients were initially identified as having a confirmed diagnosis of COVID-19 disease. The authors then evaluated the proportion of the patient group with pre-existing diabetes mellitus and the proportion of total patients receiving dexamethasone therapy. Performance of regular CBG monitoring was assessed, with particular attention to patients with a recorded $C B G>12 \mathrm{mmol} / \mathrm{L}$ triggering initiation of insulin treatment, as adapted from national guidance ${ }^{1}$ and taking into account the risks and benefits of various antidiabetic medications. ${ }^{1,2,6}$ The proportion of patients who warranted such treatment, and received it appropriately, was also assessed.

\section{Interventions}

Data were collected at regular intervals throughout the study: baseline data prior to any intervention; over the course of 2 months following a 1-week run-in from the implementation of a local 'Hyperglycaemia in COVID-19' guideline; and again, over 1 month following a 1-week run-in from the implementation of a local 'COVID-19 Acute Care Bundle'.

The initial guideline was developed by a multidisciplinary team (MDT) of the project authors, Diabetes Consultant Physicians and an inpatient Diabetes Specialist Nurse, with subsequent publication on the local clinical guidelines intranet page. Awareness of the guideline was disseminated to staff by email.

The subsequent intervention, a 'COVID-19 Acute Care Bundle', was developed later in the project by the authors in conjunction with the medical leads of the acute medical unit (AMU). Taking the form of a two-page checklist/guideline, this was part of a wider project to optimise the overall acute medical management of COVID-19 patients (including basic investigation, prescribing and escalation decisions) and included a prompt for implementing a CBG chart for a COVID-19-positive patient. Infographics regarding the management of hyperglycaemia in COVID-19 were included on the reverse of the document. The bundle was introduced at an AMU safety brief before being made available on the local intranet page and in hard copy in the AMU MDT office.

\section{Results}

One hundred and twenty hospitalised adults with COVID-19 disease were evaluated in this project over a 4-month period $(12 \%$ in phase $1,56 \%$ in phase $2,32 \%$ in phase 3 ). Of all patients studied, $24(20 \%)$ had a pre-existing diagnosis of diabetes mellitus and 74 (61.7\%) patients received dexamethasone treatment for COVID-19 disease. A total of 26 (21.7\%) patients had a CBG of $>12 \mathrm{mmol} / \mathrm{L}$ that would merit insulin treatment.

Prior to any intervention, $78.6 \%$ of patients received regular CBG monitoring; however, no patients studied received appropriate treatment for hyperglycaemia. Following the first intervention, in cycle 1,54-88\% of patients had regular CBG monitoring and $71-100 \%$ of patients received appropriate treatment for hyperglycaemia. Following implementation of the second intervention, in cycle $2,83-100 \%$ of patients received regular monitoring and $75-100 \%$ received appropriate treatment. The overall results throughout the duration of the project are shown in Figure 1.

\section{Discussion}

The prevalence of co-existing diabetes mellitus in COVID-19 disease in other studies is $17-28.3 \%, 3,7,8$ correlating with the findings of this project. Conversely, a British multicentre prospective cohort study by Närhi et al found that only $33.5 \%$ of hospitalised COVID19 patients received steroid therapy (with missing data from 18.3\% of total patients). ${ }^{9}$ This figure is significantly less than demonstrated for the population in the current study. Smaller sample size may explain some of this effect; however, given that the current study was undertaken from November 2020, considerable impact is likely due to the widely recognised findings of the RECOVERY trial, ${ }^{10}$ encouraging use of dexamethasone in COVID-19 patients. The study by Närhi et al assessed data from June to September 2020, ${ }^{9}$ when the use of dexamethasone therapy in COVID-19 was just gaining popularity.

Our study suggests that $21.7 \%$ of patients warranted management of hyperglycaemia for CBG $>12 \mathrm{mmol} / \mathrm{L}$. This compares to an American multicentre study of a patient group with diabetes and/or uncontrolled hyperglycaemia where $37.8 \%$ of patient days were spent with a mean blood glucose level $>180 \mathrm{mg} / \mathrm{dL}^{11}$ (equivalent to $10 \mathrm{mmol} / \mathrm{L}$ ). Given the morbidity associated with hyperglycaemia in COVID-19 disease, both the study by Bode et al and the current study demonstrate a significant proportion of patients requiring intervention for above-threshold hyperglycaemia.

During this study, performance with CBG recording was consistently high, even prior to intervention. This may reflect general good 
Figure 1. Monitoring and management of hyperglycaemia in patients with COVID-19 disease over time.

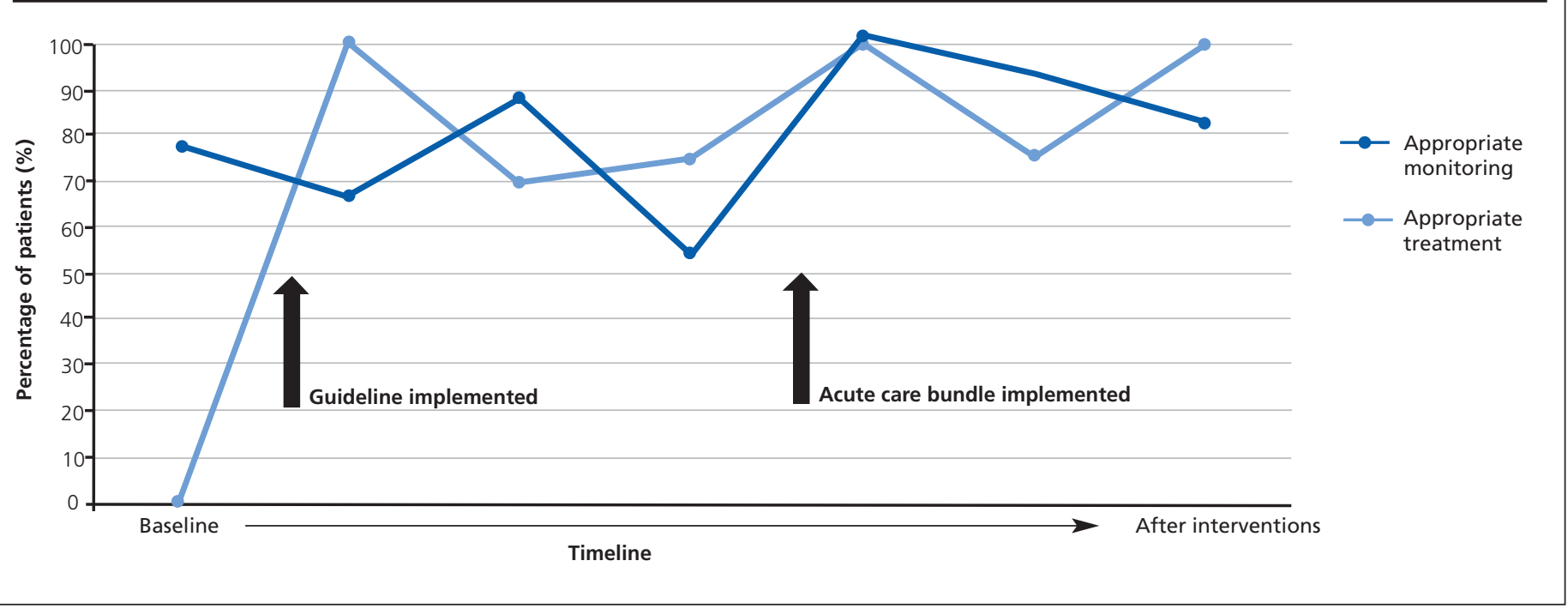

practice within the hospital and is likely supported by the proportion of people with diabetes and/or patients on dexamethasone where regular monitoring of CBG levels is already common practice. Performance with CBG monitoring over time increased following interventions. The primary outcome of this study - appropriate treatment of hyperglycaemia - also improved over time. These data demonstrated a clear improvement in appropriate therapy following the implementation of a local guideline and care bundle for COVID-19 patients. There may also be an experience effect contributing to improved performance as clinicians become more familiar with appropriate glycaemic management over time.

At the time of publication, the authors were aware of few other single-centre quality improvement projects addressing dysglycaemia in patients with COVID-19 disease. As such, this project demonstrates novel methods of tackling the issue at a local level through sustainable practical solutions. The current guideline and care bundle have since been approved at a hospital clinical governance meeting to ensure ongoing use for possible future waves of COVID19 presentations. Although the current study has differentiated patients by presence of diabetes and/or steroid therapy, future study would benefit from a larger sample size to corroborate findings within each study phase. Similarly, future study would benefit from implementation within other hospital settings, and investigation of the differences on outcomes between male and female patients, age brackets, diabetes subtypes and in those with stress glycaemia or in other acute causes of hyperglycaemia (such as pancreatic insult, intravenous fluid therapy, concurrent illness). Examination of additional outcomes such as length of stay, incidence of critical care admission, incidence of dysglycaemic complications and mortality may enhance future practice with prognostic relevance.

\section{Conclusion}

Hyperglycaemia is an important complication of COVID-19 disease, associated with increased morbidity and mortality. The presence of co-existing diabetes mellitus and/or steroid therapy in such patients

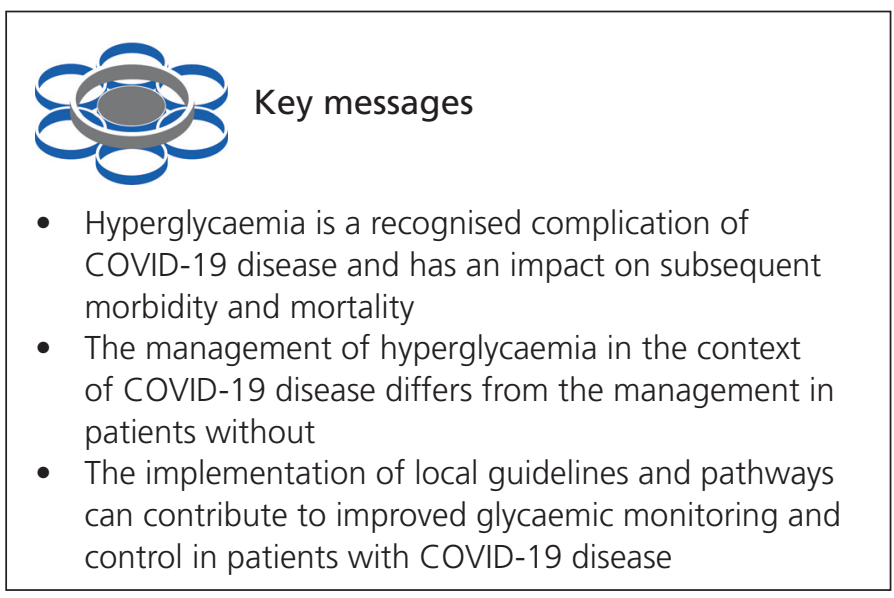

are recognised as exacerbators of this effect, and it has been previously described that early glycaemic control in the inpatient with COVID-19 disease impacts significantly on outcomes. Until further research demonstrates otherwise, there are limited safe treatment options for hyperglycaemia in patients with COVID-19 disease beyond that of insulin therapy. This quality improvement project investigated the monitoring and management of glycaemic control in 120 inpatients with COVID-19 disease in one hospital setting before, during and following two changes of practice. The implementation of a COVID-19 specific hyperglycaemia guideline and a subsequent acute care 'bundle' have contributed towards improved blood glucose monitoring and hyperglycaemia management for this patient group, although there would be much to gain from more detailed study within patient subgroups and on longer-term outcomes for future work in this area.

\section{Conflict of interest: None.}

Funding: This research did not receive any specific grant from funding agencies in the public, commercial, or not-for-profit sectors. 


\section{References}

1. Diabetes UK National Inpatient Diabetes COVID-19 Response Group. COVID: Diabetes. Dexamethasone therapy in COVID-19 patients: implications and guidance for the management of blood glucose in people with and without diabetes. Available from: https://www.diabetes.org.uk/resources-s3/public/ 2020-06/COvID_Dex_v1.4.pdf (accessed 1 April 2021).

2. Lim S, Bae JH, Kwon HS, Nauck MA. COVID-19 and diabetes mellitus: from pathophysiology to clinical management. Nat Rev Endocrinol 2021;17:1130. https://doi.org/10.1038/s41574-020-00435-4

3. Sardu C, D'Onofrio N, Balestrieri ML, et al. Hyperglycaemia on admission to hospital and COVID-19. Diabetologia 2020;63:2486-7. https://doi.org/ 10.1007/s00125-020-05216-2

4. Apicella M, Campopiano MC, Mantuano M, et al. COVID-19 in people with diabetes: understanding the reasons for worse outcomes. Lancet Diabetes Endocrinol 2020;8(9):782-92. https://doi.org/10.1016/S22138587(20)30238-2

5. Scheen A. DPP-4 inhibition and COVID-19: from initial concerns to recent expectations. Diabetes Metab 2020;47(2):101213. https://doi.org/10.1016/ j.diabet.2020.11.005

6. Lalau JD, Al-Salameh A, Hadjadj S, et al. Metformin use is associated with a reduced risk of mortality in patients with diabetes hospitalised for COVID-19. Diabetes Metab 2020;47(5):101216. https://doi.org/10.1016/ j.diabet.2020.101216
7. Grasselli G, Zangrillo A, Zanella A, et al. Baseline characteristics and outcomes of 1591 patients infected with SARS-CoV-a Admitted to ICUs of the Lombardy Region, Italy. JAMA 2020;323(16):1574-81. https://doi.org/ 10.1001/jama.2020.5394

8. Garg S, Kim L, Whitaker M, et al. Hospitalization rates and characteristics of patients hospitalized with laboratory-confirmed coronavirus disease 2019 - COVID-NET, 14 States, March 1-30, 2020. Centres for Disease Control and Prevention. MMWR 2020;69(15):458-64. https://doi.org/10.15585/ mmwr.mm6915e3

9. Närhi F, Drake T, Harrison E, et al. Delayed adoption of corticosteroids as standard of care for hypoxic patients with COVID-19 in the UK. COVID-19 Clinical Information Network 2020. Available from: https://assets.publishing.service.gov.uk/government/uploads/system/uploads/attachment_data/fil e/935215/co-cin-delayed-adoption-steroids-covid-19-patients-s0846291020.pdf (accessed 1 April 2021).

10. Horby P, Lim WS, Emberson J, et al. Dexamethasone in hospitalized patients with Covid-19. N Engl J Med 2021;384:693-702. https://doi.org/10.1056/ NEJMoa2021436

11. Bode B, Garrett V, Messler J, et al. Glycemic characteristics and clinical outcomes of COVID-19 patients hospitalized in the United States. J Diabetes Sci Technol 2020;14(4):813-21. https://doi.org/10.1177/1932296820924469 